\title{
LX. On the refraction and dispersion of gaseous compounds, and the cause of the divergence from additive relations
}

\section{Clive Cuthbertson}

To cite this article: Clive Cuthbertson (1913) LX. On the refraction and dispersion of gaseous compounds, and the cause of the divergence from additive relations, Philosophical Magazine Series 6, 25:148, 592-604, DOI: 10.1080/14786440408634196

To link to this article: http://dx.doi.org/10.1080/14786440408634196

电 Published online: 08 Jun 2010.

Submit your article to this journal $\pi$

Џll Article views: 4

Q View related articles ¿

Citing articles: 2 View citing articles $\sqsubset$ 
$\mathrm{C}_{2}=0$, that the complete solution is given by

$$
\begin{aligned}
& \mathrm{V}_{1}=\frac{\mathrm{E}}{c}\left\{(s-a) e^{-n_{1} t} \cos \left(\omega_{1} t+\alpha_{1}\right)+(s-b) e^{-n_{2} t} \cos \left(\omega_{2} t+\alpha_{2}\right)\right\}, \\
& \mathrm{V}_{2}=p_{2} a \frac{\mathrm{E}}{c}\left\{e^{-n_{1} t} \cos \left(\omega_{1} t+\beta_{1}\right)-e^{-n_{2} t} \cos \left(\omega_{2} t+\beta_{2}\right)\right\}, \\
& \mathrm{C}_{1}=-\frac{\mathrm{K}_{1} \mathrm{E}}{c}\left\{\omega_{1}(s-a) e^{-n_{1} t} \sin \left(\omega_{1} t+\gamma_{1}\right)+\omega_{2}(s-b) e^{-n_{2} t} \sin \left(\omega_{2} t+\gamma_{2}\right)\right\}, \\
& \mathrm{C}_{2}=p_{2} a \frac{\mathrm{K}_{2} \mathrm{E}}{c}\left\{-\omega_{1} e^{-n_{2} t} \sin \left(\omega_{1} t+\delta_{1}\right)+\omega_{2} e^{-n_{2} t} \sin \left(\omega_{2} t+\delta_{2}\right)\right\},
\end{aligned}
$$

where $p_{2}=\frac{\mathrm{K}_{1} \mathrm{M}}{\mathrm{K}_{2} \mathrm{~L}_{2}}=b \mathrm{~K}_{1} \mathrm{M}$ as before, and

$$
\begin{array}{ll}
\gamma_{1}=4 \frac{s-b}{c^{2} \omega_{1}} \zeta, & \gamma_{2}=-4 \frac{s-a}{c^{2} \omega_{2}} \zeta, \\
\delta_{1}=2 \frac{a-b}{c^{2} \omega_{1}} \zeta, & \delta_{2}=2 \frac{a-b}{c^{2} \omega_{2}} \zeta,
\end{array}
$$

in which $\zeta=a \lambda_{2}-b \lambda_{1}$

$$
\text { and } \quad \begin{aligned}
& \alpha_{1}=\gamma_{1}-\frac{n_{1}}{\omega_{1}}, \quad \alpha_{2}=\gamma_{2}-\frac{n_{2}}{\omega_{2}}, \\
& \beta_{1}=\delta_{1}-\frac{n_{1}}{\omega_{1}}, \quad \beta_{2}=\delta_{2}-\frac{n_{2}}{\omega_{2}} .
\end{aligned}
$$

LX. On the Refraction and Dispersion of Gaseous Compounds, and the Cause of the Divergence from Additive Relations. By Cuive Cuthbertson *.

$\mathrm{T}^{\mathrm{T}}$ is well known that the refractivities of simple gaseous 1 compounds do not follow an additive rule so closely as those of solids or liquids. The refraction equivalents of Gladstone and Dale and their followers were found to be fairly constant, at any rate for the same class of compound. But, in gases, the discrepancies observed are much wider; and this appears the more surprising since, in other fields of research, the gaseous state has proved peculiarly favourable for the discovery of simple relations.

With the object of investigating the cause of these apparent anomalies the writer has, with the help of others, measured the refraction and dispersion of a number of

* Communicated by Prof. A. W. Porter, F.R.S. 
elements and simple compounds in the gaseous state between $\lambda \lambda 6708$ and 4800 ; and, though the field of investigation is still somewhat restricted, sufficient data have now been collected to warrant the formulation of an hypothesis which seems to explain, in a qualitative way, the changes in refraction, dispersion, and absorption which are observed when gaseous compounds are formed from elements.

In the experimental work the refractivity of each substance was first determined for the green mercury line $\lambda=5461$, and reduced to a standard density, viz. that of hydrogen at $0^{\circ} \mathrm{C}$. and $760 \mathrm{~mm}$. multiplied by the ratio of the theoretical molecular weight to that of hydrogen. The dispersion was then found by measuring the refractivity at seven other wave-lengths, $\lambda \lambda 6708,6438,5790,5770,5209,5085,4800$, relatively to the value obtained for $\lambda 5461$.

The eight values of the refractivity were generally found to fall on a smooth curve, which can be fitted by an equation of Sellmeyer's form

$$
\frac{\mu^{2}-1}{2}=\mu-1 \text { (approx.) }=\frac{C}{n_{0}^{2}-n^{2}},
$$

where $\pi_{0}^{2}$ is the square of the frequency of the free vibration in the molecule (assuming there to be only one), and $n^{2}$ is the square of the frequency of the light, and is equal to

$$
\frac{\mathrm{V}^{2}}{\lambda^{2}}=\frac{9 \times 10^{20}}{\lambda^{2}} \text { (in cms.). }
$$

In the following table (p. 594) are given the values of the constants of this equation, calculated from the experimental results, for those elements and compounds which will be referred to below.

In this table column 4 gives, for convenience, the value of $\lambda_{0}$ corresponding to that of $n_{0}{ }^{2}$ in column 3 . Column 5 grives the value of $\mu-1$ for infinite waves found by equating it to $\mathrm{C} / n_{0}{ }^{2}$. Column 6 shows, in the case of compounds, the additive value of the refractivity, i.e. that obtained by adding those of its constituents. In this calculation the refractivity of a single atom of an element is taken as half that found for biatomic molecules, and similarly for polyatomic molecules.

The last column shows the difference between the additive and experimental refractivities of compounds.

The modern theory of dispersion indicates that an intimate relation exists between dispersion and absorption. The refractivity should be abnormally high on the less refrangible

Phil. Mag. S. 6. Vol. 25. No. 148. April 1913. $2 \mathrm{~S}$ 


\section{4}

Mr. Clive Cuthbertson on the

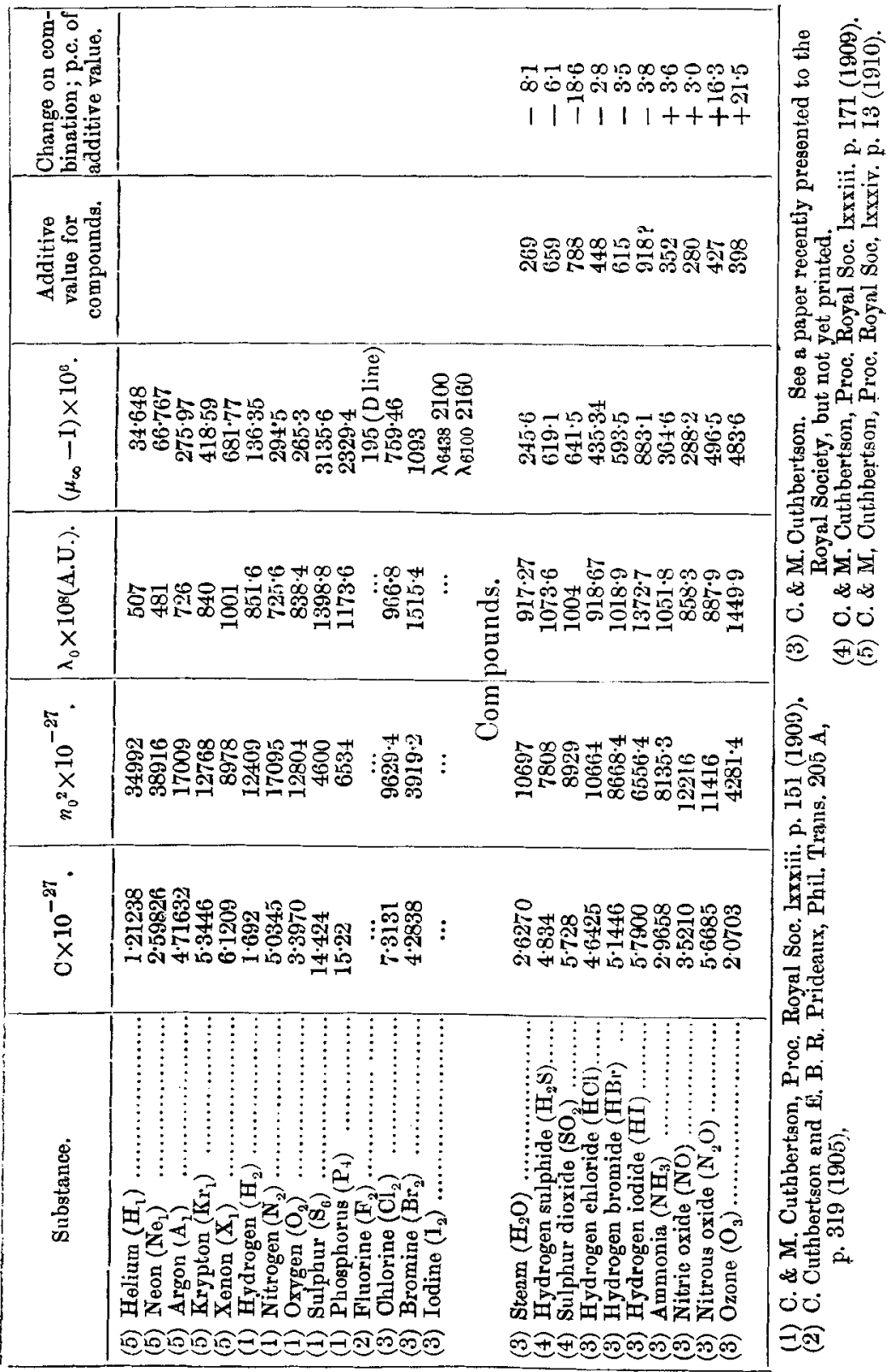


Refraction and Dispersion of Gaseous Compounds. $\quad 595$

side of an absorption band, and abnormally low on the more refrangible side. It may, therefore, be expected that the changes of position of absorption bands in the spectra of elements or compounds should corroborate the conclusions to be drawn from the variations of refraction and dispersion.

We are, thus, able to obtain three kinds of evidence from which to infer the physical meaning of the changes which take place. If, in the equation given above, $\mathrm{C}$ is altered by the act of combination, the whole scale of the figures is proportionally affected. If the free frequency is changed the value of $n_{0}^{2}$ will differ from that which it would have had on an additive hypothesis. If the absorption spectrum of the compound differs from that of its constituents, we have a clue to the identification of that portion of the refractivity which is connected with it.

The experimental results shown in the table above, and the concurrent changes in absorption, so far as they are known, may be summarized as follows:-

A. (1) In hydrochloric, hydrobromic, hydriodic acids, hydrogen disulphide, and sulphur dioxide, the refractivity of the compound is less than the sum of the refractivities of its constituents, and the dispersive power of the compound lies between those of its constituents.

(2) In the spectra of chlorine, bromine, iodine, and sulphur, strong absorption bands occur in the violet and neur ultriviolet, which disappear in the compound.

B. (1) In nitrous oxide, nitric oxide, ammonia, and ozone, the refractivity of the compound is greater than the sum of the refractivities of the constituents, and the dispersive power is greater than that of either constituent.

(2) In the specfra of nitrous oxide, nitric oxide, and ozone, absorption-bands appear in the ultra-violet which are not present in the spectra of the elements.

To explain these results I propose the following hypothesis :-

(1) That the refractivity of a gaseous element (except those whose molecules are monatomic) or compound is mainly made up of two portions which may be called the atomic and the interatomic. The former is due to vibrations of parts of the same atom relatively to one another (on the electromagnetic theory of electrons relatively to the rest of the atom). The free frequency lies in the extreme violet $(\lambda=1000$ A.U. approximately), and the dispersion curve is very flat in the visible region. The refractivity due to this 
mode of vibration is roughly $\cdot 8$ or $\cdot 9$ of the whole refractivity measured in the visible. The absorption connected with it is that which lies in the Schumann region. The atomic refractivity, being due to vibrations governed by forces which have their seat exclusively in a single atom, may be assumed to remain nearly constant. The latter, or interatomic portion of the refractivity, is the result of the existence of free periods in the visible or neighbouring portions of the ultra-violet or infra-red, due to forces which have their seat in more than one atom of the same molecule. The absorption due to these vibrations is that which is observed in the spectrum of these regions. The interatomic refractivity of an atom of an element varies with the atom with which it is combined, and with the nature of the linkage.

(2) When two elements combine to form a compound the observed changes of refraction, dispersion, and absorption are due to the disappearance of the interatomic portions of their refractivities and the appearance of new interatomic free frequencies.

An example will make the hypothesis clearer. Gaseous chlorine is characterized by high refractivity, moderately high dispersive power, and strong contiuuous absorption in the ultra-violet. Hydrogen has low refractive and dispersive power and exhibits no trace of absorption up to the limits of the Schumann region. The resulting compound ( $\mathrm{HCl}$ ) has a refractivity 2.8 per cent. less than the sum of the refractivities of $\frac{1}{2}\left(\mathrm{Cl}_{2}\right)+\frac{1}{2}\left(\mathrm{H}_{2}\right)$, a dispersive power intermediate between those of chlorine and hydrogen, and an absence of absorption, so far as is known, in the ultra-violet.

In the first place it appears highly probable that both the chlorine and the hydrogen atom carry into the compound the main portion of their refractivity. The additive rule fails, no doubt, to go further than a first approximation, even in the most favourable instances; but no one who studies the figures for gases or the work of Gladstone and Dale on solids and liquids can avoid the conviction that this rule must be the starting point of the explanation.

We have, then, to account for a small change in the sum of the refractive powers of the two elements, coupled with a considerable change in the dispersive power, which is indicated by the increase of $n_{0}{ }^{2}$ from $9629.4 \times 10^{27}$ in $\mathrm{Cl}_{2}$ to $10697 \times 10^{27}$ in $\mathrm{HCl}$, and the disappearance of an absorption band. This could be done by supposing that the refractivity of one of the elements $\left(\mathrm{Ol}_{2}\right)$ contains a term of which the absolute value is small but the slope steep, and that this term disappears when the molecule is broken up. The 
curve which expresses such a term would rise rapidly, indicating an absorption band in the near ultra-violet, as is the case in chlorine, and the disappearance of this term would be accompanied by the disappearance of the absorption band, as we find is the case when hydrochloric acid is formed.

The inference that the forces which determine this free frequency have their seat in more than one atom is based on the fact that when the link which binds the atoms of chlorine together is broken the absorption band disappears.

In the figure these changes are exhibited graphically.

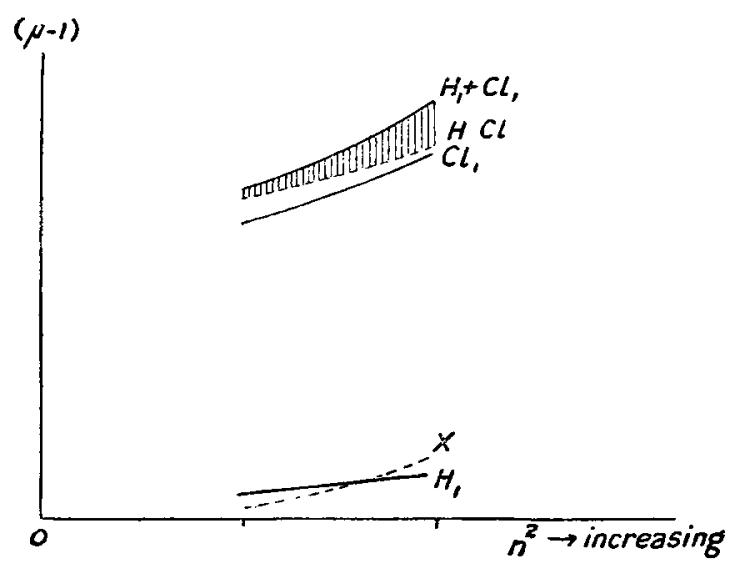

Plotting the refractivities against squares of firequencies, let $\mathrm{Cl}_{1}$ denote the refractivity of gaseous chlorine divided by two, $\mathrm{H}_{1}$ that of hydrogen divided by two, $\mathrm{H}_{1}+\mathrm{Cl}_{1}$ the additive curve, and $H(l)$ the experimental curve for hydrogen chloride. What has to be accounted for is the disappearance of the portion indicated by vertical shading between $\mathrm{H}_{1}+$ $\mathrm{Cl}_{1}$ and $\mathrm{HCl}$. Consider this portion as due to a distinct free frequency, and lay it down on the base-line as shown at $X$. It is evident that the curvature is great, since the ratio of the ordinates at the red and violet ends is greater than when the curve is bodily shifted higher, and it is natural to associate it with the absorption band which occurs in the near ultra-violet in chlorine.

It may be objected that no reason has been shown why the whole of the observed changes should be attributed to the chlorine molecule, and no allowance made for the disappearance of the interatomic refractivity of the hydrogen molecule or the appearance of a new interatomic effect for the molecule of hydrochloric acid. The criticism is just; and it must be admitted that some allowance ought to be 
made for these changes. But reasons can be advanced for assigning to them less importance than is given to the change in the chlorine molecule. In the first place, the refractivity of hydrogen is less than one-fifth of that of chlorine, so that small changes in its refractivity due to similar causes would probably be in the same proportion. The same argument applies to hydrochloric acid, whose refractivity is about onehalf that of chlorine. Again, neither hydrogen nor hydrochloric acid shows any absorption in the ultra-violet as chlorine does, so that, on the proposed hypothesis, the changes in their refraction and dispersion, due to the breaking up and formation of the molecules respectively, may also be expected to be small. Finally, the interatomic effects due to hydrogen and hydrochloric acid occur on different sides of the equation and tend to balance each other.

But the best test of the legitimacy of the hypothesis is to be found in the numerical results which are obtained from it.

The effect of the preceding analysis has been to split up the expression for the refractivity of chlorine into two parts, one of which, it is suggested, is due to the atomic and the other to the interatomic frequencies of the chlorine molecule. The values of the atomic refractivity are obtained by deducting the experimental values for $\frac{1}{2}\left(\mathrm{H}_{2}\right)$ from those for $\mathrm{HCl}$, while those for the interatomic refractivity of $\mathrm{Cl}_{2}$ are found by deducting twice the resulting figure for $\left(\mathrm{Cl}_{1}\right)$ from the experimental values for $\left(\mathrm{Cl}_{2}\right)$.

The first calculation gives eight values for the atomic refractivity, which are fitted by the curve given by

$$
(\mu-1)_{\mathrm{Cl}_{1}}=\frac{3.8787 \times 10^{27}}{10558 \times 10^{27}-n^{2}}, \quad \cdot \quad \text {. }
$$

while those for the interatomic refractivity are given by

$$
(\mu-1)_{\mathrm{Cl}_{1}-\mathrm{Cl}_{1}}=\frac{\cdot 07302 \times 10^{27}}{2935 \times 10^{27}-n^{2}} \cdot \quad . \quad .
$$

The relations of the refraction, dispersion, and absorpiion of bromine and hydrobromic acid and iodine and hydriodic acid are exactly similar to those of chlorine and hydrochloric acid.

Adopting the same procedure as that given above we obtain for the atomic refractivities of bromine and iodine:-

$$
\begin{aligned}
(\mu-1)_{\mathrm{Br}_{1}} & =\frac{4.3566 \times 10^{27}}{82 y 4 \times 10^{27}-n^{2}}, \quad . \quad . \\
(\mu-1)_{\mathrm{I}_{1}} & =\frac{5 \cdot 1548 \times 10^{27}}{6325 \times 10^{27}-n^{2}}, \quad . \quad . \quad .
\end{aligned}
$$


Refraction and Dispersion of Gaseous Compounds. 599 and for the interatomic

$$
\begin{aligned}
(\mu-1)_{\mathrm{Br}_{1}-\mathrm{Br}_{1}} & =\frac{039018 \times 10^{27}}{713.4 \times 10^{27}-n^{2}}, \ldots . \\
(\mu-1)_{\mathrm{I}_{1}-\mathrm{I}_{1}} & =\frac{.0893 \times 10^{27}}{456 \times 10^{27}-n^{2}} . .
\end{aligned}
$$

It will be noticed that the three expressions on the righthand side of $(1),(3),(4)$ form a series in which the numerators increase regularly with increasing atomic weight, and the denominators decrease. In this respect they resemble the expressions for the refractivity of the three inert gases argon, krypton, and xenon which I published two years ago* ; and, if the numerators of the six expressions are compared together by pairs, it is found that the numbers bear the same proportion to each other.

Comparison of the values of $\mathrm{C}$ in the expression $\mu-1=\mathrm{C}_{1} / n_{0}^{2}-n^{2}$ for the halogens and inert gases.

$$
\begin{aligned}
& \mathrm{C}^{\prime} / \mathrm{C}^{\prime \prime} \text {. Ratio. } \\
& \frac{\text { Chlorine }}{\text { Argon }}=\frac{3 \cdot 8787}{4 \cdot 7163}=\cdot 822 \text {, } \\
& \frac{\text { Bromine }}{\text { Krypton }}=\frac{4 \cdot 3566}{5 \cdot 3446}=\cdot 815 \text {, } \\
& \frac{\text { Iodine }}{\text { Xenon }}=\frac{5 \cdot 1548}{6 \cdot 1209}=\cdot 842 \text {. }
\end{aligned}
$$

Since the numerical values of the refractivities of each triad are in the ratio of 2,3 , and 5 approximately, it follows that the denominators of the same formulæ, when compared in the same manner by pairs, bear approximately the same ratio.

Comparison of the values of $n_{0}^{2}$ for the halogens and inert gases.

$$
\begin{aligned}
& \text { Chlorine } \\
& \begin{array}{l}
n_{0}^{\prime}{ }_{0}^{2} / n^{\prime \prime}{ }_{0}^{2} . \\
\text { Argon }
\end{array}=\frac{10558}{17009}=\cdot 621, \\
& \text { Bromine } \\
& \frac{8294}{\text { Krypton }}=\frac{829}{12768}=\cdot 650, \\
& \frac{\text { Iodine }}{\text { Xenon }}=\frac{6325}{8978}=\cdot 705 .
\end{aligned}
$$

* Phil, Mag. January 1911, p. 69. 
The parallelism between these two sets of figures is unmistakable, and seems to afford strong evidence that this method of obtaining expressions for the refractivity of the halogen atoms is on the right track.

But it is possible to carry the comparison a step further. In the paper in the Phil. Mag. just referred to it was shown that, in the case of the four inert gases whose critical temperature has been determined, there is a constant ratio between the squares of the numerator of the dispersion formulæ (which, on Drude's theory, are proportional to the number of electrons in the atom which influence dispersion) and the critical temperatures. It, therefore, occurred to me to compare the critical temperatures of the halogens with those of the inert gases. Unfortunately, the determinations for bromine and iodine rest on the authority of only one observer; but, assuming their accuracy, the proportionality is evident.

Comparison of the critical temperatures of the halogens and inert gases.

\begin{tabular}{|c|c|c|c|}
\hline Element. & $\underset{\text { Absolute }}{\mathbf{T}_{c}}$ & Ratio. & Observer. \\
\hline $\begin{array}{l}\text { Chlorine... } \\
\text { Argon ...... }\end{array}$ & $\left.\begin{array}{l}420 \\
155 \cdot 6\end{array}\right\}$ & 270 & $\begin{array}{l}\text { Knietsch (419), Ladenberg (421). } \\
\text { Ramsay and Travers. }\end{array}$ \\
\hline $\begin{array}{l}\text { Bromine... } \\
\text { Krypton... }\end{array}$ & $\left.\begin{array}{l}575 \\
210 \cdot 5\end{array}\right\}$ & $2 \cdot 73$ & $\begin{array}{l}\text { Nadeschdine. } \\
\text { R. \& T. }\end{array}$ \\
\hline $\begin{array}{l}\text { Iodine } \\
\text { Xenon }\end{array}$ & $\left.\begin{array}{l}785 \\
289 \cdot 6\end{array}\right\}$ & $2 \cdot 71$ & $\begin{array}{l}\text { Radice. } \\
\text { Patterson, Cripps \& Whytlaw-Gray. }\end{array}$ \\
\hline
\end{tabular}

It follows that there is a constant ratio between the squares of the numerators of the expressions for the refractivities of the halogen atoms and their critical temperatures.

Turning now to the expressions assumed to be connected with the interatomic free frequencies (2), (5), (6), we can test the usefulness of the hypothesis by comparing the position of the calculated free frequency with that of the centre of the absorption band. The coincidences are not so striking as in the case just discussed, but, considering that we are here dealing with figures which are the differences between two nearly equal quantities and that the calculation takes no account of the influence of absorption on the value of the free frequency, the agreement is not unsatisfactory.

It will be observed that, in each case, the calculated free frequency is greater than the observed, and this is also true in the case of ozone and of nitrous oxide, nitric oxide, and even of oxygen, so far as can be judged without having observed the actual centre of the absorption-band of the latter element. It seems probable that the dispersion curve 
Refraction and Disper'sion of Gaseous Compounds.

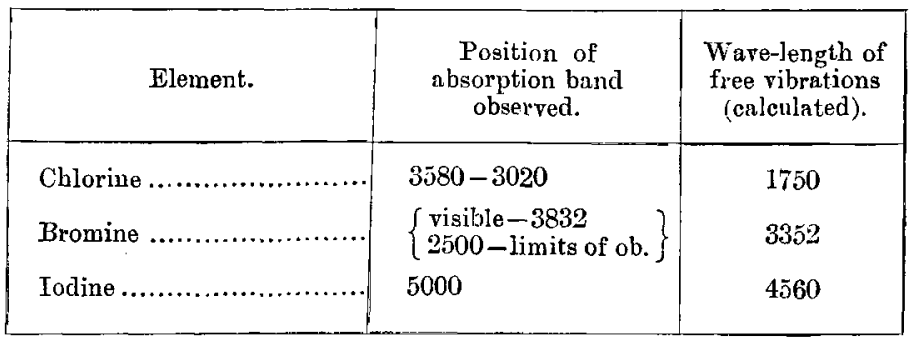

begins to be flatter than that which the simple theory demands a long way from the free frequency. It is unfortunate that we have no determinations of the coefficients of absorption in the case of these gases, by the use of which a closer approximation might be obtained.

In the case of the sulphur compound $\mathrm{H}_{2} \mathrm{~S}$ and $\mathrm{SO}_{2}$ we find that the evidence points in the same direction. Here, also, we bave an element of high refractive, dispersive, and absorptive power united to one of low refractive and dispersive power, and free from absorption; and here again the resulting compound shows a decrease of refraction, a dispersive power intermediate between those of the two components, and a disappearance of the absorption band characteristic of the sulphur molecule. The same hypothesis as in the case of the halogens will account for the direction of these changes; but it must be admitted that it is not so successful in accounting for their magnitude. The expressions which I obtain for the atomic refraction of one atom of sulphur are

$$
\frac{3.4168 \times 10^{27}}{7078 \times 10^{27}-n^{2}} \text { and } \frac{2.7780 \times 10^{27}}{7384 \times 10^{27}-n^{2}},
$$

when calculated from $\mathrm{H}_{2} \mathrm{~S}$ and $\mathrm{SO}_{2}$ respectively. But there is room for many important disturbing factors in the complexity of the molecules both of sulphur and of the compounds. A comparison of the cases of $\mathrm{NO}, \mathrm{N}_{2} \mathrm{O}$ and $\mathrm{NH}_{3}$ shows that the number of atoms in the molecule has a considerable effect on the interatomic portion of the refractivity.

Turning now to the group of compounds in which the refractivity is higher than the sum of the refractivities of its constituents, we observe that they are composed of elements like hydrogen, nitrogen, and oxygen whose refractive and dispersive power is small, so that it might be expected, on our hypothesis, that, when their molecules break up, their loss of these powers would be slight, while, when they unite 
into larger or less stable molecules, the new free frequencies introduced between the atoms of the compound would be slower than those between the atoms of the elements, with a correspondingly greater effect on the dispersion.

It should be noted that, in these cases, the only practicable test of our hypothesis is the converse of that which gives such promising results in the case of the halogens. There we neglected the interatomic refractivities of one element and the compound, and obtained a value for the atomic refractivity of the other element. Here we neglect the interatomic refractivities of both elements and obtain a value for the interatomic refractivity of the compound, and this, being the difference between two nearly equal quantities, is liable to much larger errors than the atomic.

In this group the most interesting results are obtained in the case of ozone. Here the refractivity is $21 \frac{1}{2}$ per cent. higher than the value for three atoms of oxygen, and the dispersive power is much greater. If we deduct from the experimental values for the refractivity of ozone one and a half times the refractivity of oxygen, we obtain the following result:-

$$
\lambda \times 10^{8} \text {. }
$$

\begin{tabular}{|l|c|c|c|}
\hline & 6708 & 5461 & 4800 \\
\hline$[\mu-1]_{\mathrm{O}_{3}} \times 10^{8} \ldots$ & 50764 & 52000 & 53290 \\
$3[\mu-1]_{0_{2}} \times 10^{8}$. & $\underline{40428}$ & $\underline{40755}$ & $\underline{41049}$ \\
$\dot{2}[\mu \underline{11245}$ & 12241 \\
\hline
\end{tabular}

The figures in the last line, which represent the increase in refractivity due to the interatomic effect in ozone, are fitted by the curve given by

$$
[\mu-1] \underset{\mathrm{O}_{3}-\mathrm{O}_{3}}{\left[0^{8}\right.}=\frac{\cdot 12660 \times 10^{27}}{1424.8 \times 10^{27}-n^{2}} .
$$

The wave-length which corresponds with $n_{0}{ }^{2}=1424: 8 \times 10^{27}$ is $\lambda=2520$. The absorption spectrum of ozone has been most recently measured by Meyer (Drude's Ann. xii. p. 849 (1903)), who found a maximum of absorption at $\lambda 2580$.

One other fact with regard to this substance is worth mention. If we calculate the constants of the expression for the refractivity of ozone from the values given in the first line above for $\lambda 6708$ and $\lambda 4800$, we obtain a curve 
Refraction and Dispersion of Gaseous Compounds. 603 which gives a value of $52080 \times 10^{-8}$ at $\lambda=5461$, instead of 52000 found experimentally. Hence the dispersion curve of ozone has greater curvature than can possibly be explained on the hypothesis of a single free frequency. At least two are required. The same effect was observed in a less degree in the case of chlorine.

For the three nitrogen compounds, ammonia, nitrous oxide, and nitric oxide, the refractivity of the compound is in excess and the dispersion curve becomes much steeper, suggesting the appearance of new free frequencies in the ultra-violet. But in the case of nitrous oxide the absorption spectrum has, apparently, not been examined. Nitric oxide has a band which begins about 3000 A.U. and increases to the limits of observation (Kreusler). The calculated band falls at $\lambda=1940$. Ammonia is said by Soret to show an absorption band in the ultra-violet. but Hartley denies this.

The case of steam is a difficulty on the hypothesis now advanced ; for though the dispersion curve has greater curvature than those of hydrogen and oxygen, the refractivity is notably less. This conjunction of changes is not impossible under the hypothesis, but it is less easily explicable.

In concluding this paper I wish to emphasize the fact that quantitative verification of the hypothesis is not claimed. The data are still too scanty, the range of observation too short, the gaps, both in mathematical theory and in observation, too many to permit this. All that it is desired to maintain is that the direction of the changes of refractivity, dispersion, and absorption consequent on the formation of compounds are consistent with the hypothesis that in every gaseous element (except those whose molecule is monatomic) and compound the refractivity is made up mainly of two portions. Of these one is governed by a free frequency in the far ultra-violet and is responsible for the bulk of the refractive power. Since it is always associated with the presence of an atom, it may be supposed to be due to forces which have their seat within the atom, and are hardly at all affected by neighbouring atoms. The other is due to a free frequency governed by forces which have their seat in more than one atom of the molecule, and which change with the linkage.

I think the numerical results obtained by the adoption of this hypothesis in the case of the simplest series of compounds known, the halogens and halogen acids, is strongly in its favour; and that the agreement between the observed and calculated positions of the absorption band in ozone is very remarkable. 


\section{Dr. H. Geiger and Mr. E. Marsden on the Laws of}

But whatever value is attached to the numerical evidence by those competent to judge, the broad conclusion from the direction of the changes is unaffected.

I should like also to reply to one criticism which has been made. It is not necessary to the hypothesis that the atomic free frequency should be absolutely invariable throughout all chemical changes. If it were the case that a slight change (say 1 per cent.) in the atomic free frequency would account for all the observed changes of refractivity and dispersion, the criticism would have force. But any one who examines the figures in the table for (e.g.) hydrogen, nitrogen, and ammonia, will see that no slight change in a frequency can possibly account for the observed changes. For hydrogen $n_{0}^{2} \times 10^{-27}=12409$, for nitrogen 17095 . For ammonia the number which expresses its average value is 8135 , an enormous drop. At the same time the refractivity has gone up only $3 \frac{1}{2}$ per cent. But if the number of vibrators had remained constant while the average free frequency decreased, the increase of the refractivity must have been much greater than this. Hence, to account for the observed changes, one must also assume that the number of vibrators (? electrons) has fallen off in about the same proportion as the frequency.

These two hypotheses seem much more improbable than that which is here put forward.

LXI. The Laws of Deflexion of a Particles through Large Angles*. By Dr. H. Geiger and E. Marsden $\dagger$.

TN a former papert one of us has shown that in the 1 passage of $\alpha$ particles through matter the deflexions are, on the average, small and of the order of a few degrees only. In the experiments a narrow pencil of $\alpha$ particles fell on a zinc-sulphide screen in vacuum, and the distribution of the scintillations on the screen was observed when different metal foils were placed in the path of the $a$ particles. From the distribution obtained, the most probable angle of scattering could be deduced, and it was shown that the results. could be explained on the assumption that the deflexion of a single $\alpha$ particle is the resultant of a large number of very small deflexions caused by the passage of the $\alpha_{n}$ particle through the successive individual atoms of the scattering substance.

* Communicated to $k$. d.-k. Akad. d. Wiss. Wien.

+ Communicated by Prof. E. Rutherford, F.R.S.

$\ddagger$ H. Geiger, Roy. Soc. Proc. rol. Ixxxiii. p. 492 (1910); vol. lxxxvi. p. 235 (1912). 